\title{
Associations between adherence to MIND diet and severity, duration and frequency of migraine headaches among migraine patients
}

\author{
Moein Askarpour ${ }^{1,2}$, Habib yarizadeh ${ }^{1}$, Ali Sheikhi ${ }^{1}$, Faezeh Khorsha ${ }^{1}$ and Khadijeh Mirzaei ${ }^{{ }^{*}}$ (i)
}

\begin{abstract}
Objectives: Migraine is a neurological disorder causing unbearable pain. Dietary approach is proposed as a preventive way of reducing the severity of migraine headaches. The present study aimed to examine the association between MIND diet and migraine headaches.

Results: We found that participants with higher score of MIND diet compared to those with lower score, were less likely to have severe headaches $(\mathrm{OR}=0.64 ; 95 \% \mathrm{Cl} 0.45,0.91 ; P=0.01)$. Moreover, our results showed an inversed correlation between mind diet score and duration $(\beta=-0.14,95 \% \mathrm{Cl}-1.42,-0.14, P=0.04)$ and frequency of headaches $(\beta=-0.13,95 \% \mathrm{Cl}-0.99,-0.07, P=0.03)$.
\end{abstract}

Keywords: Migraine headache, Mind diet, Visual analog scale, Migraine Disability Assessment Questionnaire

\section{Introduction}

Migraine is a common neurovascular brain disorder that often causes throbbing headaches of varying intensity, lasting hours to days [1]. It is often accompanied by complications such as vomiting and photophobia [2]. Migraine is estimated to affect $10 \%$ of the global population, more prevalent in women than men [3]. The disability caused by migraine is substantial and could impose a great economic burden upon societies [4]. Moreover, Migraineurs are at increased risk of cardiovascular disorders and mortality [5]. Therefore, finding the best approach to control and manage this disease is of utmost importance. Though the exact mechanism behind this disease is not fully clear, changes in the blood flow of

\footnotetext{
*Correspondence: mina_mirzaei101@yahoo.com

${ }^{1}$ Department of Community Nutrition, School of Nutritional Sciences and Dietetics, Tehran University of Medical Sciences (TUMS), P.O. Box:

14155-6117, Tehran, Iran

Full list of author information is available at the end of the article
}

the brain are considered to be the main stimuli. Several medications have been introduced to alleviate the symptoms [6], but given the major side effects that these medications may create [7], identifying disease-modifying risk factors to prevent headaches is crucially important. Research has shown that nutrition may have a role.

With the population of elderly growing, the rate of age-related diseases like dementia is expected to rise, making it a high-priority problem. Though there is no effective treatment for dementia, recent research has shown that the Mediterranean and Dietary Approaches to Stop Hypertension (DASH) diets could be efficacious in curtailing cognitive decline. Recently, a hybrid of these two dietary patterns, named the MIND diet, has been developed by Morris et al. [8]. The MIND diet focuses on natural plant-based foods and limits the intake of animal and high saturated fat foods. It also emphasizes the consumption of berries and green leafy vegetables which are known to have antioxidant and anti-inflammatory properties and inhibit neurotoxic death. The beneficial effect 
of the MIND diet on Alzheimer's dementia [8], depressive symptoms [9], and parkinsonian signs [10] have shown in previous studies. Though studies examining the effect of MIND diet on cognitive function are a handful, they have shown that adherence to the MIND diet was associated with slower rates of cognitive decline [11]. Since previous studies have linked migraine to inflammation and oxidative stress [12-14], we propose this hypothesis that the MIND diet could have a potential beneficial effect on migraine.

To our best knowledge, no study has ever investigated the relationship between the MIND diet and migraine, we decided to conduct this cross-sectional observational study to examine the effect of MIND diet on intensity, duration and frequency of migraine headaches.

\section{Main text}

\section{Methods}

\section{Participants}

In this cross-sectional study, 266 women aged 18-50 with history of migraine headaches were selected from two neurology clinics in Tehran. The sample size was computed according to the following formula:

$$
\mathrm{n}=\left(\left(\left[\left(\mathrm{Z}_{1-\alpha}+\mathrm{Z}_{1-\beta}\right) \times \sqrt{ } 1-\mathrm{r}^{2}\right] / \mathrm{r}\right)^{2}+2\right),
$$

which $r=0.25, \beta=0.95$, and $\alpha=0.05$ [15]. Based on this formula, 241 individuals were required; however, due to the availability of data and to account for any possible exclusion, 266 individuals were included.
In the present study, Inclusion criteria were the following: participants with history of migraine headaches diagnosed by an expert neurologist, voluntarily involvement, BMI ranged between 18.5 and 30 , and visiting the clinics for the first time. Consent forms were collected by all of the participants.

\section{MIND diet assessment}

Dietary intake was evaluated by a face-to-face interview using a 147-items semi-quantitative food frequency questionnaire (FFQ), which its reliability and validity had been already approved in Iran [16]. To compute the MIND diet scores, some dietary components of the FFQ was used in this study which are presented in Table 1 . Of 15 dietary factors in the original scoring of MIND diet, 10 of them were defined as brain healthy food groups (green leafy vegetables, other vegetables, nuts, berries, beans, whole grains, fish, poultry, olive oil, and wine) and 5 as brain unhealthy food groups (red meats, butter and stick margarine, cheese, pastries and sweets, and fried/ fast food) [11]. However, in the current study we used a modified MIND diet scoring based on Iranian food habits [9]. In this scoring, wine consumption was not considered (forbidden drinking). The other 14 food groups were applied in the MIND scoring. First, individuals were categorized according to tertile categories of dietary intakes. Participants in the bottom tertile of brain healthy food groups were given the score of 0 , those in the middle tertile were given the score of 0.5 and individuals in the top tertile were given the score of 1 . On the other hand, we

Table 1 Components of MIND diet

\begin{tabular}{|c|c|}
\hline \multicolumn{2}{|l|}{ Brain healthy foods } \\
\hline Green leafy vegetables & Greens, lettuce \\
\hline Other vegetables & $\begin{array}{l}\text { Cabbage, raw carrot, potato, peas or lima beans, green/red } \\
\text { peppers, eggplant, onion, cucumber, tomatoes, tomato } \\
\text { sauce }\end{array}$ \\
\hline Berries & Strawberries (strawberry, fresh berries) \\
\hline Nuts & Walnuts, almonds, peanuts, pistachios, hazelnuts \\
\hline Whole grains & Dark bread (Iranian) \\
\hline Fish (not fried) & Fish \\
\hline Beans & Beans, mung bean, peas, lentils, chickpea \\
\hline Poultry (not fried) & Chicken \\
\hline \multicolumn{2}{|l|}{ Brain unhealthy foods } \\
\hline Butter, margarine & Butter, margarine, animal fats \\
\hline Cheese & Cheese \\
\hline Red meat and products & Red meat, hamburger, sausages \\
\hline Fast fried foods & French fries, pizza \\
\hline Pastries and sweets & $\begin{array}{l}\text { Biscuit, ice cream, cake, confections, cocoa, Gaz (an Iranian } \\
\text { confectionery made of sugar, nuts and tamarisk), choco- } \\
\text { late }\end{array}$ \\
\hline
\end{tabular}


did vice versa about brain unhealthy food groups. Finally, the overall score was computed by summing up of all dietary components scores. Therefore, the participant's MIND diet score ranged from 0 to 14 .

\section{MIDAS and VAS questionnaires}

To assess headache-related disability, the Migraine Disability Assessment (MIDAS) questionnaire was applied. The MIDAS questionnaire validity and reliability already were assessed in Iranian population [17]. The VAS score cut-off points ranged from 1 to 10 and categorized pain severity in three levels: 1 to 3 (mild pain), 4 to 7 (moderate pain) and 8 to 10 (severe pain) [18].

\section{Assessment of other variables}

Weight was measured via a digital scale (SECA, Hamburg, Germany) to the nearest $0.1 \mathrm{~kg}$, while wearing no shoes and one layer of clothing. To examine physical activity (PA), International Physical Activity Questionnaire (IPAC), was used [19]. PA was shown as metabolic equivalent hours per week (METs h/week).

\section{Statistical analyses}

The Chi-square test was used to assess the association between the quartiles of MIND diet and qualitative variables. In addition, one-way analysis of variance (ANOVA) was used to assess the association between the quartiles of MIND diet and quantitative variables. Covariance analysis (ANCOVA) was applied to compare the dietary intakes of participants among quartiles of MIND diet by adjusting the effect of total energy. Confounding factors including total energy, age, BMI, PA, smoking status, water intake, salt intake, medication usage, and menstruation headaches were applied in adjusted models. To assess the association between the MIND score quartiles and the headache severity and disability, multinomial logistic regression model was used in crude and adjusted models. Moreover, to determine the association between headache duration and frequency (dependent variable) and the quartiles of MIND diet (independent variables), Linear Regression Models analysis was used, in crude and adjusted models. $\mathrm{P}<0.05$ was considered as level of statistically significance. SPSS version 24 (SPSS Inc., Chicago, IL, USA) was used to perform statistical analysis.

\section{Results}

\section{Study population characteristics}

The baseline characteristics of the participants among quartiles of MIND diet are shown in Table 2. The mean $( \pm \mathrm{SD})$ age, height, weight, BMI and physical activity of participants were $34.32(7.86)$ years, $1.61(0.05) \mathrm{m}, 69.41$ (13.02) kg, $26.50(4.88) \mathrm{kg} / \mathrm{m}^{2}, 407.73$ (519.13) MET/min/ week, respectively. Quantitative and qualitative variables across MIND diet quartiles did not show any significant differences. The percentages of study population among the MIND diet quartiles were: $\mathrm{Q}_{1} 27.4, \mathrm{Q}_{2} 25.5, \mathrm{Q}_{3} 25.5$, and $\mathrm{Q}_{4}$ 21.6.

\section{Dietary intake and MIND diet quartiles}

Dietary intakes of the participants among MIND diet quartiles are presented in Additional file 1: Table S1. Participants in the highest quartile of MIND diet had higher consumption of vegetables, legumes and nut, while had lower intake of refined grain and energy intake compared to the lowest quartile of MIND diet score. However, the consumption of fruits, dairy, whole grain, meat, and water intake across quartiles of MIND diet did not show any significant differences.

\section{MIND diet and migraine headache}

The association between duration, frequency, severity and disability of migraine headache across quartiles of MIND diet in crude model and adjusted model are shown in Table 3. In the crude model of multinomial logistic regression, greatest adherence to the MIND diet did not show a significant relation with severity of disability based on MIDAS score (OR $=0.97$; $95 \%$ CI 0.58 , 1.52; $P=0.93$ ). Even after adjustment for potential confounding factors including energy intake, age, BMI, PA, smoking status, water intake, salt intake, menstruation headaches, and medication usage, no association was observed $(\mathrm{OR}=0.96 ; 95 \%$ CI $0.59,1.49 ; P=0.81)$. A significant inverse association was observed between MIND diet score and odds of severe headaches in crude model (OR $=0.69 ; 95 \%$ CI $0.47,0.98 ; P=0.04)$. After adjustment for the potential confounding factors, individuals in the highest quartile of MIND diet were $36 \%$ less likely to have severe headaches $(\mathrm{OR}=0.64 ; 95 \% \mathrm{CI} 0.45,0.91 ; P=0.01)$ compared with those in the lowest quartile.

Linear Regression Models analysis (LRM) showed an inverse association between MIND diet score and duration of migraine headaches in crude $(\beta=-0.16,95 \% \mathrm{CI}$ $-1.43,-0.31 P=0.01)$, and adjusted models $(\beta=-0.14$, $95 \%$ CI $-1.42,-0.14, P=0.04)$. Moreover, frequency of headaches indicated an indirect correlation with MIND diet score in crude model $(\beta=-0.14,95 \%$ CI -1.01 , $-0.12, P=0.02)$, and adjusted model $(\beta=-0.13,95 \% \mathrm{CI}$ $-0.99,-0.07, P=0.03)$.

\section{Discussion}

To the best of our knowledge, the present study is the first to examine the association between the modified MIND diet and migraine headaches. The main analyses showed that higher adherence to the modified MIND diet is related to lower headache intensity, duration, and frequency among migraine patients. 
Table 2 General characteristics of study population among quartiles (Q) of MIND diet score

\begin{tabular}{|c|c|c|c|c|c|c|}
\hline & \multicolumn{5}{|c|}{ Quartiles of the MIND diet score } & \multirow[b]{2}{*}{ *P-value } \\
\hline & Total $(\mathrm{N}=266)$ & $\begin{array}{l}Q_{1}(n=73) \\
a_{4.12}\end{array}$ & $\begin{array}{l}\mathrm{Q}_{2}(\mathrm{n}=68) \\
{ }^{\mathrm{a}} 6.14\end{array}$ & $\begin{array}{l}\mathrm{Q}_{3}(\mathrm{n}=68) \\
\mathrm{a}_{8.07}\end{array}$ & $\begin{array}{l}\mathrm{Q}_{4}(\mathrm{n}=57) \\
\mathrm{a}_{10.21}\end{array}$ & \\
\hline Age (years) & $34.32 \pm 7.86$ & $34.57 \pm 8.43$ & $34.21 \pm 8.17$ & $34.55 \pm 6.69$ & $33.71 \pm 8.24$ & 0.87 \\
\hline Height (m) & $1.61 \pm 0.05$ & $1.62 \pm 0.05$ & $1.62 \pm 0.05$ & $1.61 \pm 0.05$ & $1.62 \pm 0.04$ & 0.48 \\
\hline Weight (kg) & $69.41 \pm 13.02$ & $67.34 \pm 13.47$ & $70.27 \pm 13.57$ & $68.57 \pm 12.12$ & $72.27 \pm 13.28$ & 0.27 \\
\hline BMI $\left(\mathrm{kg} / \mathrm{m}^{2}\right)$ & $26.50 \pm 4.88$ & $25.87 \pm 4.73$ & $26.97 \pm 5.45$ & $26.18 \pm 4.86$ & $27.38 \pm 4.56$ & 0.29 \\
\hline PA (MET-h/week) & $407.73 \pm 519.13$ & $410.73 \pm 724.27$ & $449.41 \pm 410.83$ & $412.02 \pm 420.24$ & $452.25 \pm 471.23$ & 0.78 \\
\hline \multicolumn{6}{|c|}{ Current smoker (n (\%)) } & 0.11 \\
\hline Yes & $13(4.9)$ & $5(1.9)$ & $0(0)$ & $3(1.1)$ & $5(1.9)$ & \\
\hline No & $253(95.1)$ & $68(25.5)$ & $68(25.5)$ & $65(24.1)$ & $52(20)$ & \\
\hline \multicolumn{6}{|c|}{ Marital status (n (\%)) } & 0.75 \\
\hline Single & $74(27.8)$ & $21(7.9)$ & $20(7.5)$ & $16(6)$ & $17(6.4)$ & \\
\hline Married & $192(72.2)$ & $52(19.5)$ & $48(18.1)$ & $52(19.5)$ & $40(15.1)$ & \\
\hline \multicolumn{6}{|c|}{ Education status (n (\%)) } & 0.87 \\
\hline$\leq$ Diploma & $104(39.1)$ & $30(12.3)$ & $26(9.7)$ & $24(9.1)$ & $24(9.1)$ & \\
\hline > Diploma & $162(60.9)$ & $43(16.1)$ & $42(15.6)$ & $44(16.5)$ & $33(12.7)$ & \\
\hline \multicolumn{6}{|c|}{ Medication use (n (\%)) } & 0.27 \\
\hline Yes & $127(47.7)$ & $32(12.1)$ & $28(10.5)$ & $37(13.9)$ & $30(11.2)$ & \\
\hline No & $139(52.3)$ & $41(15.4)$ & $40(15)$ & $31(11.7)$ & $27(10.2)$ & \\
\hline \multicolumn{6}{|c|}{ Family history of migraine $(\mathrm{n}(\%))$} & 0.73 \\
\hline Yes & $173(65)$ & $51(19.1)$ & $45(16.9)$ & $45(16.9)$ & $33(12.1)$ & \\
\hline No & $92(35)$ & $22(8.4)$ & $23(8.7)$ & $23(8.7)$ & $24(9.2)$ & \\
\hline
\end{tabular}

Quantitative variables (age, height, weight, BMI, PA, headache duration) reported as Mean $\pm \mathrm{SD}$

Qualitative variables (current smoker, education and marital) reported as number (\%)

$B M I$ body mass index, $P A$ physical activity

${ }^{*}$ Chi-square test and ANOVA were applied for qualitative and quantitative variables, respectively

a Median MIND diet score

Table 3 Crude and multivariable-adjusted odds ratios for severity, disability, duration and frequency of migraine headaches across MIND diet scores

\begin{tabular}{|c|c|c|c|c|c|c|}
\hline & \multicolumn{2}{|c|}{ Crude models } & \multirow[t]{2}{*}{$P$-value } & \multicolumn{2}{|c|}{ Adjusted models } & \multirow[t]{2}{*}{${ }^{\mathbf{b}} P$-value } \\
\hline & OR & $(0.95 \% \mathrm{Cl})$ & & OR & $(0.95 \% \mathrm{Cl})$ & \\
\hline \multicolumn{7}{|l|}{ VAS } \\
\hline Mild pain ${ }^{c}$ & - & - & - & - & - & - \\
\hline Moderate pain & 0.81 & $(0.61,1.12)$ & 0.21 & 0.85 & $(0.65,1.14)$ & 0.28 \\
\hline Sever pain & 0.69 & $(0.47,0.98)$ & 0.04 & 0.64 & $(0.45,0.91)$ & 0.01 \\
\hline \multicolumn{7}{|l|}{ MIDAS } \\
\hline Without disabilityc & - & - & - & - & - & - \\
\hline Mild disability & 0.91 & $(0.57,1.24)$ & 0.72 & 0.93 & $(0.61,1.27)$ & 0.77 \\
\hline Moderate disability & 0.90 & $(0.57,1.27)$ & 0.68 & 0.85 & $(0.54,1.19)$ & 0.28 \\
\hline Severe disability & 0.96 & $(0.59,1.49)$ & 0.81 & 0.97 & $(0.58,1.52)$ & 0.93 \\
\hline Headache duration of each attack & ${ }^{a}-0.16$ & $(-1.43,-0.31)$ & 0.01 & ${ }^{a}-0.14$ & $(-1.42,-0.14)$ & 0.04 \\
\hline Frequency of headache in month & ${ }^{a}-0.14$ & $(-1.01,-0.12)$ & 0.02 & $\mathrm{a}-0.13$ & $(-0.99,-0.07)$ & 0.03 \\
\hline
\end{tabular}

MIDAS Migraine Disability Assessment Questionnaire, VAS visual analog scale

a The $\beta$ coefficient has been shown

b Adjusted for confounders

c Considered as reference group 
However, the MIDAS score, which assesses the disability caused by migraine, did not have a significant relation with the modified MIND diet.

There are some possible mechanisms through which the effect of the MIND diet on migraine could be explained. Mainstream medicine views migraine headaches as a result of the excitation of sensory fibers in the brain due to neurogenic inflammation which happens in response to even normal stimuli. The notion of migraine being a neuroinflammatory disease has been strengthened by studies investigating neuropeptides such as calcitonin gene-related peptide (CGRP). CGRP is known to be involved in several physiological processes including the dilation of cerebral and dural blood vessels and the release of inflammatory mediators [20]. Now that the current evidence points out that CGRP plays a central role in the pathophysiology of migraine, blocking the effects of it seems to be a logical clinical approach, as anti-CGRP drugs administered for migraine, such as Triptan, have proven fairly successful in attenuating headaches [21]. Dietary antioxidants could also impose similar effects if consumed in adequate amounts. One study demonstrated that rats orally fed with grape seed extract for 2 weeks had lower expression of CGRP than control rats [22]. Moreover, dietary components in vegetables such as Indole-3-carbinol and sulforaphane could inhibit the activation of CGRP receptor signaling [23]. Mediterranean and DASH diets have long been associated with lower levels of oxidative stress and inflammation [24-26]. The antioxidants in berries and the vitamin E in olive oil, green leafy vegetables and nuts could ease migraine by protecting the brain from oxidative stress [27]. Additionally, the omega-3 fatty acids found in fish may benefit migraine by lowering inflammation in the brain [28].

Low magnesium level has been attributed to causing cerebrovascular constriction and increased vascular reactivity and membrane receptor activity to mediators, producing headaches. Accordingly, several studies have shown that migraineurs usually have low brain magnesium levels during migraine attacks and may also suffer from a magnesium deficiency $[29,30]$. It is worth noting that certain food items (such as green leafy vegetables, nuts, and seeds, legumes) which are integral parts of the MIND diet, are rich sources of magnesium.

Several studies are suggesting a positive association between hypertension and migraine [31-33]. Malignant hypertension, through imposing pressure on cranium, could be responsible for causing headaches. Research showing the effect of DASH and Mediterranean diets on lowering blood pressure is abundant [15].

\section{Conclusion}

We found that higher adherence to the modified MIND diet may reduce the severity of pain in migraine patients. These initial findings need to be confirmed with prospective studies in order to confirm the relationship between the modified MIND diet and migraine.

\section{Limitation}

The number of enlisted subjects were relatively low. The absence of men is another major limitation that is worth noting. The cross-sectional design of the study prohibits us from inferring causality. Finally, questionnaire responses are subjectively based on participant's memory and their perception of pain.

\section{Supplementary information}

Supplementary information accompanies this paper at https://doi. org/10.1186/s13104-020-05181-4.

Additional file 1: Table S1. Dietary intake of study population among quartiles $(Q)$ of PRAL and NEAP.

\section{Abbreviations}

ANOVA: One-way analysis of variance; FFQ: Food frequency questionnaire; MIDAS: Migraine Disability Assessment; IPAC: International Physical Activity Questionnaire; PA: Physical activity; LRM: Linear Regression Models; TUMS:

Tehran University of Medical Science; VAS: Visual analog scale.

\section{Acknowledgements}

The authors thank the directors of the school of Nutritional and Dietetics at Tehran University of medical sciences for allowing them to conduct a comparative cross-sectional study for the purpose of evaluating migraine pain. This study was supported by Grants (ID: 95-03-161-33142 and 96-01-161-34479) from Tehran University of Medical Sciences.

\section{Authors' contributions}

$M A$, and HY designed research; ASH conducted research; KhM and MA analyzed data; ASH and FK wrote the paper; KhM had primary responsibility for final content. All authors read and approved the final manuscript.

Funding

Funding was provided by Tehran University of Medical Sciences.

Availability of data and materials

The data are not publicly available due to containing information that could compromise the privacy of research participants.

\section{Ethics approval and consent to participate}

All participants signed a written informed consent and the study was approved by Tehran University of Medical Sciences ethics committee (IR. TUMS.VCR.REC.1395.1597)

Consent for publication

Not applicable.

\section{Competing interests}

All of authors declared that they have no competing interests.

\section{Author details}

${ }^{1}$ Department of Community Nutrition, School of Nutritional Sciences and Dietetics, Tehran University of Medical Sciences (TUMS), P.O. Box: 14155-6117, 
Tehran, Iran. ${ }^{2}$ Students' Scientific Research Center (SSRC), Tehran University of Medical Sciences (TUMS), Tehran, Iran.

\section{Received: 28 April 2020 Accepted: 10 July 2020}

Published online: 16 July 2020

\section{References}

1. Lyngberg AC, Rasmussen BK, Jørgensen T, Jensen R. Has the prevalence of migraine and tension-type headache changed over a 12-year period? A Danish population survey. Eur J Epidemiol. 2005;20(3):243-9.

2. Steiner TJ, Stovner L, Vos T. GBD 2015: migraine is the third cause of disability in under 50s. Berlin: Springer; 2016.

3. Woldeamanuel YW, Cowan RP. Migraine affects 1 in 10 people worldwide featuring recent rise: a systematic review and meta-analysis of community-based studies involving 6 million participants. J Neurol Sci. 2017;372:307-15.

4. Goldberg LD. The cost of migraine and its treatment. Am J Manage Care. 2005;11(2 Suppl):S62-7.

5. Chen D, Willis-Parker M, Lundberg GP. Migraine headache: is it only a neurological disorder? Links between migraine and cardiovascular disorders. Trends Cardiovasc Med. 2019. https://doi.org/10.1016/j.tcm.2019.10.005.

6. Pardutz A, Schoenen J. NSAIDs in the acute treatment of migraine: a review of clinical and experimental data. Pharmaceuticals. 2010;3(6):1966-87.

7. Grosser T, Ricciotti E, FitzGerald GA. The cardiovascular pharmacology of nonsteroidal anti-inflammatory drugs. Trends Pharmacol Sci. 2017;38(8):733-48.

8. Morris MC, Tangney CC, Wang Y, Sacks FM, Bennett DA, Aggarwal NT. MIND diet associated with reduced incidence of Alzheimer's disease. Alzheimer's Dement. 2015;11(9):1007-14.

9. Salari-Moghaddam A, Keshteli AH, Mousavi SM, Afshar H, Esmaillzadeh A, Adibi P. Adherence to the MIND diet and prevalence of psychological disorders in adults. J Affect Disord. 2019;256:96-102.

10. Agarwal P, Wang Y, Buchman A, Holland T, Bennett D, Morris M. MIND diet associated with reduced incidence and delayed progression of Parkinsonism in old age. J Nutr Health Aging. 2018;22(10):1211-5.

11. Morris MC, Tangney CC, Wang Y, Sacks FM, Barnes LL, Bennett DA, Aggarwal NT. MIND diet slows cognitive decline with aging. Alzheimer's Dement. 2015;11(9):1015-22.

12. Peres MFP, Del Rio MS, Seabra M, Tufik S, Abucham J, Cipolla-Neto J, Silberstein S, Zukerman E. Hypothalamic involvement in chronic migraine. J Neurol Neurosurg Psychiatry. 2001;71(6):747-51.

13. Juhasz G, ZsombokT, Gonda X, Nagyne N, Modosne E, Bagdy G. Effects of autogenic training on nitroglycerin-induced headaches. Headache J Head Face Pain. 2007;47(3):371-83

14. Williamson DJ, Hargreaves RJ. Neurogenic inflammation in the context of migraine. Microsc Res Tech. 2001;53(3):167-78.

15. Mirzababaei A, Khorsha F, Togha M, Yekaninejad MS, Okhovat AA, Mirzaei K. Associations between adherence to dietary approaches to stop hypertension (DASH) diet and migraine headache severity and duration among women. Nutr Neurosci. 2020;23(5):335-42.

16. Mirmiran P, Hosseini-Esfahani F, Jessri M, Mahan LK, Shiva N, Azizi F. Does dietary intake by Tehranian adults align with the 2005 dietary guidelines for Americans? Observations from the Tehran lipid and glucose study. J Health Popul Nutr. 2011;29(1):39.
17. Ghorbani A, Chitsaz A. Comparison of validity and reliability of the Migraine disability assessment (MIDAS) versus headache impact test (HIT) in an Iranian population. Iran J Neurol. 2011;10(3-4):39.

18. Price DD, McGrath PA, Rafii A, Buckingham B. The validation of visual analogue scales as ratio scale measures for chronic and experimental pain. Pain. 1983;17(1):45-56.

19. Wanner M, Probst-Hensch N, Kriemler S, Meier F, Autenrieth C, Martin BW. Validation of the long international physical activity questionnaire: influence of age and language region. Prev Med Rep. 2016;3:250-6.

20. Malhotra R. Understanding migraine: potential role of neurogenic inflammation. Ann Indian Acad Neurol. 2016;19(2):175-82.

21. Benemei S, Cortese F, Labastida-Ramírez A, Marchese F, Pellesi L, Romoli M, Vollesen AL, Lampl C, Ashina M, School of Advanced Studies of the European Headache F. Triptans and CGRP blockade-impact on the cranial vasculature. J Headache Pain. 2017;18(1):103.

22. Cady RJ, Hirst JJ, Durham PL. Dietary grape seed polyphenols repress neuron and glia activation in trigeminal ganglion and trigeminal nucleus caudalis. Mol Pain. 2010;6:91.

23. Jain MM, Kumari N, Rai G. A novel formulation of veggies with potent anti-migraine activity. Int J Comput Biol Drug Des. 2015;8(1):54-61.

24. Fito M, Guxens M, Corella D, Saez G, Estruch R, de la Torre R, Frances F, Cabezas C, Lopez-Sabater MDC, Marrugat J, et al. Effect of a traditional Mediterranean diet on lipoprotein oxidation: a randomized controlled trial. Arch Intern Med. 2007;167(11):1195-203.

25. Chrysohoou C, Panagiotakos DB, Pitsavos C, Das UN, Stefanadis C. Adherence to the Mediterranean diet attenuates inflammation and coagulation process in healthy adults: the ATTICA Study. J Am Coll Cardiol. 2004;44(1):152-8

26. Lopes HF, Martin KL, Nashar K, Morrow JD, Goodfriend TL, Egan BM. DASH diet lowers blood pressure and lipid-induced oxidative stress in obesity. Hypertension (Dallas, Tex: 1979). 2003;41(3):422-30.

27. Gómez-Pinilla F. Brain foods: the effects of nutrients on brain function. Nat Rev Neurosci. 2008;9(7):568-78.

28. Devassy JG, Leng S, Gabbs M, Monirujjaman M, Aukema HM. Omega-3 polyunsaturated fatty acids and oxylipins in neuroinflammation and management of alzheimer disease. Adv Nutr. 2016;7(5):905-16.

29. Mauskop A, Altura BM. Role of magnesium in the pathogenesis and treatment of migraines. Clin Neurosci. 1998;5(1):24-7.

30. Ramadan NM, Halvorson H, Vande-Linde A, Levine SR, Helpern JA, Welch KM. Low brain magnesium in migraine. Headache. 1989;29(7):416-9.

31. Cirillo M, Stellato D, Lombardi C, De Santo NG, Covelli V. Headache and cardiovascular risk factors: positive association with hypertension. Headache. 1999;39(6):409-16.

32. Gudmundsson LS, Thorgeirsson G, Sigfusson N, Sigvaldason H, Johannsson M. Migraine patients have lower systolic but higher diastolic blood pressure compared with controls in a population-based study of 21,537 subjects. The Reykjavik study. Cephalalgia Int J Headache. 2006;26(4):436-44.

33. Franceschi $\mathrm{M}$, Colombo B, Rossi $\mathrm{P}$, Canal $\mathrm{N}$. Headache in a populationbased elderly cohort. An ancillary study to the Italian Longitudinal Study of Aging (ILSA). Headache. 1997;37(2):79-82.

\section{Publisher's Note}

Springer Nature remains neutral with regard to jurisdictional claims in published maps and institutional affiliations. 